\title{
The eDoctor: Effective Use of Mobile Technology to Advance Care and Learning
}

\section{Cheng-Kai Kao" ${ }^{1 *}$, Vineet M Arora', Roger $\mathrm{Yu}^{2}{ }^{2}$, Amit Pahwa ${ }^{3}$, Anoop Agrawal ${ }^{4}$ and Anuj K Dalal ${ }^{5}$}

${ }^{1}$ Department of Medicine, University of Chicago, Chicago, IL, USA

${ }^{2}$ Department of Medicine, Scripps Clinic, San Diego, CA, USA

${ }^{3}$ Department of Medicine, The Johns Hopkins Hospital, Baltimore, MD, USA

${ }^{4}$ Department of Medicine, Baylor College of Medicine, Houston, TX, USA

${ }^{5}$ Department of Medicine, Brigham and Women's Hospital, Boston, MA, USA

\begin{abstract}
Frontline clinicians are often faced with the task of rapidly sifting through a high volume of information from a variety of electronic sources. Managing this information deluge can be overwhelming, yet paramount to the delivery of highvalue healthcare. Thus, today's clinicians must become the 'e-Doctors', who can best leverage mobile technology to overcome the informational challenges at point-of-care. In this review, we provide a framework for clinicians to manage electronic information, and go through a day in the life of an e-Doctor to understand how clinicians may use mobile health apps to accomplish different tasks and enhance the care and learning.
\end{abstract}

Keywords: Mobile technology; Mobile health; Mobile devices; Smartphone applications; Information management

\section{Introduction}

In this mobile era, it is estimated over $80 \%$ of physicians use smartphones or tablet computers in their daily practice [1]. While mobile devices are great companions, they can be equally problematicwork disruptions from excessive notifications are not uncommon and can lead to serious adverse events [2]. Furthermore, healthcare providers now have access to a bevy of mobile healthcare applications or "mHealth apps" that can be used at the point-of-care to facilitate a variety of tasks (Table 1). However, with over 40,000 mHealth apps [3,4] and lack of supervising authorities [5] it is challenging to know how to best utilize these apps. Therefore, today's doctors must not only be compassionate healthcare providers, but also become an "eDoctor," who can skilfully leverage mobile technology to provide timely, high-value healthcare at point-of-care. In this article, we will shadow Dr. Mobile to understand how clinicians may overcome different informational challenges encountered daily in the hospital setting.

\section{Mobile Technology to Advance Care and Learning}

While driving to the hospital, Dr. Mobile notes that his smartphone has been constantly pinging. After arrival, he takes out the phone and finds more than 20 new email alerts and mobile app notifications.

In many professions, including healthcare providers, the task of managing constant influx of new information can be overwhelming $[6,7]$. Increasingly, much of the information is being "pushed" to them via the Internet, often as an email delivered to their mobile device. In order to manage incoming information, the clinicians should have a basic understanding of the how information is transacted over the Internet (Box 1 for discussion on "Push" and "Pull"), and then can better organize and prioritize information in mobile devices. For example, eDoctors may employ a "push" strategy to be notified of highpriority information (e.g., a significantly abnormal test result), while using "pull" strategy to retrieve less-urgent information (e.g., a newly published article in the field of interest) - this should minimize alert fatigue that would otherwise occur if all information was constantly pushed.

After Dr. Mobile configures his smartphone to selectively push or pull different types of information, he goes to see his new patient, a 76 year-old non-English speaking female with history of COPD presenting with a fever and shortness of breath. After a detailed history taking and physical exams using phone-based interpretation, Dr. Mobile walks to the workstation to write orders but suddenly realizes he forgets to ask about allergy history. About 5-9\% of Americans report their English-speaking ability as "not at all" or "not well" [8]. Furthermore, comprehension of health-related information can be much worse than perceived by clinicians $[8,9]$. Patients with limited English proficiency have a drastically higher rate of adverse events and death compared with their English-speaking counterparts [10]. Although hospitals and clinics are increasing access to interpreter services (e.g., interpreters, phone-based language services) to assist clinical staff, these services are not always available at a convenient time, not to mention the availability of interpreters for less commonly spoken languages is much less.

Detailed medical history taking should only be performed with a certified medical interpreter, which can be in-person or via a video remote interpreting service on mobile devices. For simple, focused questions with expected straightforward answers, medical translation apps can provide timely efficient translation at the point-of-care. These apps typically have a large off-line database of categorized, commonlyasked questions for users to download, and are capable of both reading the questions and showing the text, which can be helpful for patients who are hard of hearing. Use cases may include asking simple questions (e.g., "Do you have any allergy to medications?"), giving straightforward instruction (e.g., "Please point to where you are having pain."), notifying before action (e.g., "I am going to listen to your heart and lung."), and showing the text in the patient's native language for hard-hearing patients.

The patient reports no allergies after listening to the question from the translation app. Dr. Mobile then orders a chest X-ray, antibiotics

*Corresponding author: Cheng-Kai Kao, MD, Department of Medicine, University of Chicago, 5841 South Maryland Avenue, MC-5000, W314, Chicago, IL, USA, Tel: (773) 834-8129; Fax (773) 795-7398; E-mail: ckkao@medicine.bsd.uchicago.edu

Received June 26, 2017; Accepted July 03, 2017; Published July 05, 2017

Citation: Kao CK, Arora VM, Yu R, Pahwa A, Agrawal A, et al. (2017) The eDoctor: Effective Use of Mobile Technology to Advance Care and Learning. J Health Med Informat 8: 270. doi: 10.4172/2157-7420.1000270

Copyright: ( 2017 Kao CK, et al. This is an open-access article distributed under the terms of the Creative Commons Attribution License, which permits unrestricted use, distribution, and reproduction in any medium, provided the original author and source are credited. 


\begin{tabular}{|c|c|c|}
\hline Category & Examples & Use Case \\
\hline Medical Translation & Canopy Translator, MediBabble & Ask the patient to point to the source of pain \\
\hline Drug Reference & Epocrates, Micromedex & Look up side effects of a medication \\
\hline Medical Calculator & Calculate by QxMD & $\begin{array}{l}\text { Calculate CHA2DS2-VASc score to determine annual risk of stroke in patients } \\
\text { with atrial fibrillation }\end{array}$ \\
\hline Patient Education \& Engagement & $\begin{array}{l}\text { Use Inhalers (Asthma/COPD), MediSafe (Pill } \\
\text { reminder) }\end{array}$ & Set up reminders for elderly patients with multiple medications \\
\hline Bedside Education & BaiBoard HD (digital whiteboard), DrawMD series & $\begin{array}{l}\text { Illustrate the cascade of coagulation on tablets when teaching about direct Xa } \\
\text { inhibitors }\end{array}$ \\
\hline Literature Review & Read by QxMD, Docphin & Read the personalized medical journal based on areas of interest \\
\hline Social Media \& Networking & Twitter, Facebook, Doximity, QuantiaMD, Sermo & Share an inspiring speech or idea at a conference \\
\hline Secure Messaging & Doximity, TigerText & $\begin{array}{l}\text { Communicate with other care team members such as residents regarding } \\
\text { patient disposition }\end{array}$ \\
\hline Mobile Electronic Health Record & Epic Haiku and Canto & Review lab results and notes on the go \\
\hline
\end{tabular}

Table 1: Categories and examples of mHealth apps.

"Push" is a style of Internet-based communication in which the request for the information transaction is initiated by the web-server. In contrast, with "Pull" the request is initiated by the client (e.g., a mobile email app). Mobile users may encounter the terms "push" and "fetch" (or "pull") when configuring email on their mobile device. When "push" is selected, the web-server will automatically deliver new emails to the client and notify the user. If "fetch" is selected, the client checks the web-server periodically to identify new emails and then downloads them. In essence, by using "fetch," the user "pulls" information from the web-server on-demand or at certain time intervals. Additionally, many mobile devices allow users to configure notifications for individual apps-users may selectively turn on or off "push" notifications. When notifications are turned off, the user must manually access the mobile app to fetch (or "pull") content.

Box 1: Push vs. pull strategies for email and mobile apps.

and nebulizers. Later in the day, the patient's son brings from home the patient's pills in an unlabelled bottle.

With the growing number of prescription and non-prescription medications, keeping track of multiple trade names and generic counterparts has become increasingly challenging. Drug reference apps, the most commonly used app by physicians [11], have fundamentally changed how we perform these tasks. They allow physicians to identify pills by shape, size, and colour, learn more about potential adverse reactions, check for drug-drug interactions among multiple medications, calculate adjusted doses by weight and renal function, and provide cost information at the point of care. Increasingly, these apps connect with a broad range of formularies including discounted drug lists from national chain pharmacies. By having access to these formularies at the point of care, clinicians can select the most appropriate medication within a drug class to minimize out-of-pocket costs for patients.

Dr. Mobile successfully identifies the pills using drug reference apps. When Dr. Mobile asks about the patient's home condition, the son and only caregiver reports he sometimes forgets to give the patient medications and has been confused about how to use the inhalers appropriately.

Approximately $50 \%$ of patients do not take medications as prescribed, and poor compliance is one of the leading causes of medication-related hospitalizations [12,13]. Meanwhile, almost $65 \%$ of Americans own smartphones, and $65 \%$ of those who own smartphones have used them for health-related purposes [14]. The potential for improving medication non-adherence using smartphones is considerable. For example, patients can download a pill reminder app or create recurring calendar events to help patients remember to take their medications at specific times.

Likewise, the opportunity for delivering patient education in realtime is becoming increasingly apparent. For example, over $80 \%$ of patients with COPD misuse their inhaler, 15 but those who are taught extensively are less likely to use acute health care services within the first 30 days after discharge; however, the effects of the intervention wane over time $[15,16]$. As a patient-education tool, a mobile app can reinforce instructions provided at discharge. In this case, Dr. Mobile may suggest a specific mobile app that helps the patient identify different types of inhalers and demonstrates how to use them appropriately. Although the impact of mobile apps on patient activation and self-management has not been extensively studied, early evidence is encouraging. For example, apps that coach diabetes patients how to manage their point-of-care glucose levels have demonstrated a reduction in HbAlc [17]. Thus, patient education apps can be a venue to advance patient education and engagement, and improve health outcomes.

The son decides to use the mobile apps Dr. Mobile recommends to help care for his mother. In the afternoon, a group of medical students show up for bedside teaching rounds. Dr. Mobile wants to discuss the common pathogens for community-acquired pneumonia and review a relevant article.

Bedside teaching rounds in the modern era pose numerous challenges for academic physicians. Duty-hour regulations, interruptions from clinical activities, and infection control protocols have placed constraints on the time available to teach [18]. Advocates for bedside teaching have stressed the need to be pro-active and prepared. One strategy is to create "teaching scripts" or mini-lectures that target clinical pearls and medical decision-making [19]. However, memorizing and recalling such scripts can become burdensome. Moreover, an oral discussion can be insufficient to relay complex concepts and engage learners.

Bedside education apps on tablet computers offer unique solutions and advantages for bedside teaching rounds. Some offer original teaching content that can be used immediately, while digital whiteboard apps provide educators a good platform to integrate customized content including images, algorithms, tables, and articles in one place, along with annotation ability that can be handy during teaching. Common scenarios include providing more depth for specific teaching points or addressing the learner's question more suitably. These teachable moments often require a specific image or article that can be accessed on the go [20]. These apps also enable educators to develop innovative teaching methods such as creating a multimedia quiz that engages learners (Figure 1) [21]. These mobile educational technologies enhance learner experience and engagement, and help overcome time limitations during the dynamic clinical work with prepared teaching content [22]. 
Citation: Kao CK, Arora VM, Yu R, Pahwa A, Agrawal A, et al. (2017) The eDoctor: Effective Use of Mobile Technology to Advance Care and Learning. J Health Med Informat 8: 270. doi: 10.4172/2157-7420.1000270

Page 3 of 4

After an enriching interactive teaching round with medical students, Dr. Mobile wants to review the latest research related to his field of medicine, but finds that there are too many new articles to keep up with.

Staying up-to-date on the medical literature is a challenge for any clinician, but has become easier through the use of literature review apps on mobile devices. These apps typically aggregate abstracts, summaries of articles, and newsfeeds from medical journals and trusted websites. They provide quick access to full-text articles from medical journals. In contrast to accessing medical journals from your institution's online library through a Virtual Private Network (VPN), after initial log-on and authentication, usernames and passwords can be stored in the app for quicker entry on subsequent visits. These apps typically ask users to identify their specific interests (e.g., mobile health, quality improvement, etc.) during the initial signup process; the app will then automatically retrieve articles from relevant peer-reviewed journals in the areas of interest (e.g., Journal of Mobile Technology in Medicine). Users may have the option to "like" or "dislike" specific articles of interest allowing users to further refine and personalize their articles preferences. In essence, these apps adapt and learn the user's article preferences, retrieving more relevant articles over time.

After reviewing the latest updates in medicine, Dr. Mobile wants to share his thoughts about what he has learned with other colleague.

Social media has revolutionized how clinicians can share and learn information. Social networking and micro blogging websites such as

Facebook and Twitter can be powerful tools for clinicians to share and learn information and recent events among professional and personal networks. While Facebook is often used to share information with friends and family, it can also be used to receive information from professional societies by "liking" those pages. Similarly, clinicians can obtain information on Twitter by directly following specific individuals and/or groups of interests (e.g., Journal of Mobile Technology in Medicine (@JAMAInternalMed)). Twitter also allows users to pull relevant information through the use of indexed "hashtags" that search recent tweets related to a specific topic of interest. For example, clinicians may conduct a search using the hash tagged term "\#health IT" to retrieve the latest tweets related to the topic. Similarly, clinicians can type one or more hashtags while composing a tweet so that other users may search and retrieve their tweets.

\section{Discussion}

The exponential growth of mHealth app development has provided numerous tools that clinicians may use on their mobile devices. However, the accuracy, quality and usability of the mHealth apps have not been thoroughly established. Currently, the Food and Drug Administration (FDA) only regulates mHealth apps that are used as an accessory to an FDA-regulated medical device or transform a mobile platform into a regulated medical device [23] (e.g., an app that turns a smartphone into an ECG machine)-the review and certification of most of the other mHealth apps are left largely to the marketplace. Calls for

C

A

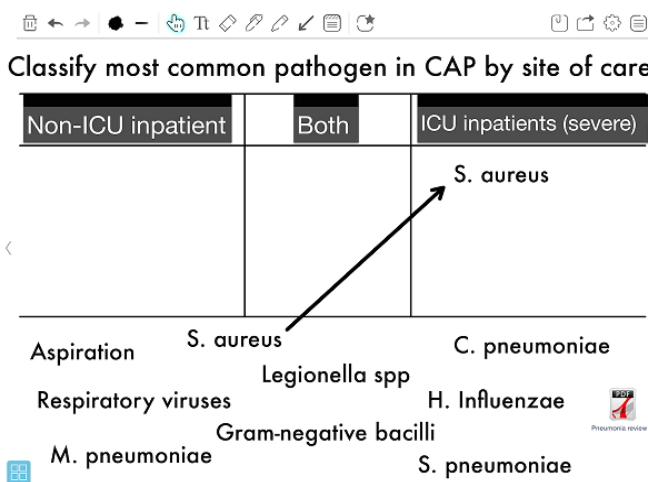

B

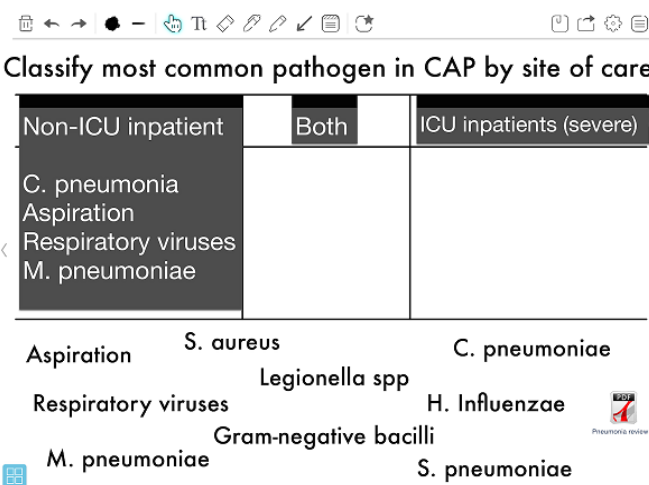

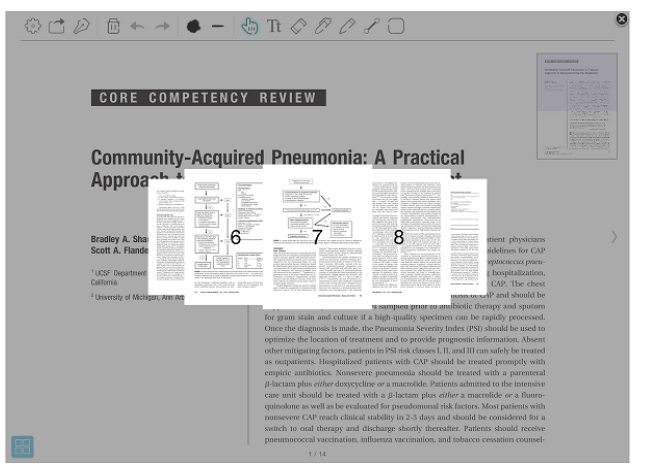

D

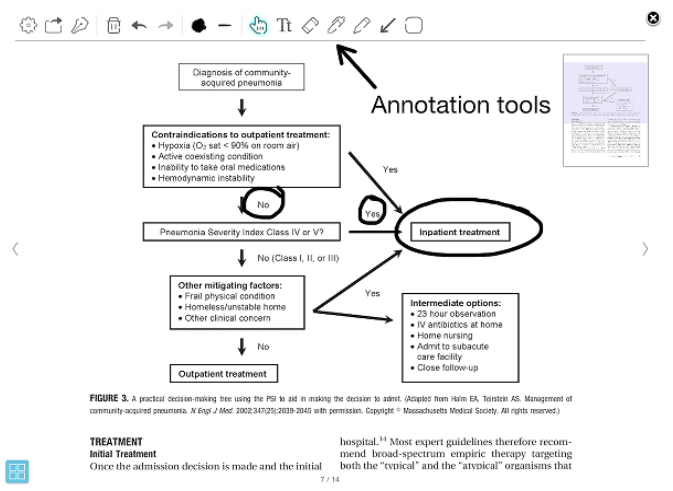

Figure 1: Example use of bedside education app (made by BaiBoard HD app). (A) Learners engage in an exercise of classifying pathogens to appropriate category by dragging names using their finger across the screen as depicted by the arrow. (B) Answers can be revealed by built-in drop down windows. PDF of reference article is embedded in bottom right handed corner of screen for quick access. (C) Selecting the PDF opens article in carousel view for easy browsing to find page of interest. (D) Selected page of PDF can be annotated, resized and shared via e-mail. 
Citation: Kao CK, Arora VM, Yu R, Pahwa A, Agrawal A, et al. (2017) The eDoctor: Effective Use of Mobile Technology to Advance Care and Learning. J Health Med Informat 8: 270. doi: 10.4172/2157-7420.1000270

Page 4 of 4

\begin{tabular}{|l|l|}
\hline Policy Considerations & Example \\
\hline Governance & Define the structure of the mobile techonology governance \\
\hline Device ownership & Determine the mobile devices should be 'corporate-owned, personally enabled' (COPE) or 'bring your own device' (BYOD) \\
\hline Mobile device etiquette & Promote the bedside manner of using mobile devices at point of care \\
\hline Privacy & $\begin{array}{l}\text { Illustrate the standard protocols of handling protected health information, including secure messaging and image upload and } \\
\text { storage }\end{array}$ \\
\hline Security & $\begin{array}{l}\text { Illustrate the standard protocols of device encryption, user authentication, system updates, and remote device wipe if lost or } \\
\text { stolen }\end{array}$ \\
\hline App review & Consider providing a list of trusted mHealth apps with a regular review process \\
\hline
\end{tabular}

Table 2: Policy considerations of best practices for mobile devices.

rigorous "app certification" criteria and unbiased accrediting bodies have been made [5], but actual attempts have been challenging due to the large volume of available apps and the complexity of the certification process $[24,25]$. Last but not least, more research is needed to evaluate the clinical efficacy and cost effectiveness of mHealth apps [26].

Given the increased use of mobile technology in the healthcare environment, we recommend the healthcare professionals to create and maintain a policy regarding the best practices of using mobile devices in their practice. The major considerations for the policy are listed in Table 2.

\section{Conclusion}

Managing information on the go is becoming essential to delivering timely, high-value healthcare in this modern world. Mobile technology is a powerful tool that can be used by doctors to optimize information management, augment day-to-day clinical tasks, enhance patient communication and education, advance medical knowledge, and facilitate scholarly conversation. With refined integration of mobile technology into daily practice, eDoctors will be able to enhance the efficacy, efficiency, and safety of their care and learning.

\section{References}

1. Wolters Kluwer Health (2013) Physician outlook survey

2. Halamka J (2013) Order interrupted by text: multitasking mishap. AORN J 98: 115.

3. http://www.research2guidance.com/r2g/research2guidance-mHealth-AppDeveloper-Economics-2014.pdf

4. West DM (2016) How mobile devices are transforming healthcare.

5. Powell AC, Landman AB, Bates DW (2014) IN search of a few good apps. JAMA 311: 1851-1852

6. Hemp P (2016) Death by information overload.

7. Hemp P (2016) Managing information overload.

8. Bureau UC (2011) Language use in the United States.

9. http://www.ahrq.gov/sites/default/files/publications/files/lepguide.pdf

10. "What Did the Doctor Say? (2016)" Improving health literacy to protect patien safety.

11. Conn J (2013) No longer a novelty, medical apps are increasingly valuable to clinicians and patients. Mod Healthc 43: 16-18.
12. Osterberg L, Blaschke T (2005) Adherence to medication. N Engl J Med 353: 487-497.

13. lihara N, Tsukamoto T, Morita S, Miyoshi C, Takabatake K, et al. (2004) Beliefs of chronically ill Japanese patients that lead to intentional non-adherence to medication. J Clin Pharm Ther 29: 417-424.

14. Smith A (2015) U.S. Smartphone Use in 2015.

15. Press VG, Arora VM, Shah LM, Lewis SL, Ivy K, et al. (2011) Misuse of respiratory inhalers in hospitalized patients with asthma or COPD. J Gen Intern Med 26: 635-642.

16. Press VG, Arora V, Constantine KL, Naureckas ET, White SR, et al. (2014) Forget me not: a randomized trial of the durability of hospital-based education on inhalers for patients with COPD or asthma. J Gen Intern Med 29: S102-S102.

17. Quinn CC, Shardell MD, Terrin ML, Barr EA, Ballew SH, et al. (2011) Clusterrandomized trial of a mobile phone personalized behavioral intervention for blood glucose control. Diabetes Care 34: 1934-1942.

18. McGee S (2014) Bedside teaching rounds reconsidered. JAMA 311: 1971-1972.

19. Abdool MA, Bradley D (2013) Twelve tips to improve medical teaching rounds Med Teach 35: 895-899.

20. Harrison R, Allen E (2006) Teaching internal medicine residents in the new era J Gen Intern Med 21: 447-452.

21. Sharpe BA, Flanders SA (2006) Community-acquired pneumonia: A practical approach to management for the hospitalist. J Hosp Med 1: 177-190.

22. (2016) Effective use of educational technology in medical education.

23. U.S. Food and Drug Administration (2016) Mobile medical applications: guidance for industry and food and drug administration staff.

24. Misra S (2014) Happtique's recent setback shows that health app certification is a flawed proposition.

25. Chan SR, Misra S (2014) Certification of mobile apps for health care. JAMA 312: $1155-1156$

26. Boulos MNK, Brewer AC, Karimkhani C, Buller DB, Dellavalle RP (2014) Mobile medical and health apps: state of the art, concerns, regulatory control and certification. Online J Public Health Inform 5: 229. 